\title{
ATP-Binding Cassette Expression Profilling Revealed its Role in Development and Regulating Stress Response in Potato (Solanum Tuberosum)
}

madiha zaynab ( $\square$ madiha.zaynab14@gmail.com )

Shenzhen University https://orcid.org/0000-0002-2999-9896

Athar Hussain

University of Management and Technology

Mateen Sajid

Ghazi University

Muhammad Ahsan

Islamia University: The Islamia University of Bahawalpur Pakistan

Yasir Sharif

Fujian Agriculture and Forestry University

Mahpara Fatima

Fujian Agriculture and Forestry University

Muhammad Ammar Raza

King Khalid University

Khalid Ali Khan

King Khalid University

Shuangfei Li

Shenzhen University

Research Article

Keywords: ATP-binding cassette (ABC), Solanum tuberosum, RT-qPCR

Posted Date: July 26th, 2021

DOI: https://doi.org/10.21203/rs.3.rs-715478/v1

License: (-) (1) This work is licensed under a Creative Commons Attribution 4.0 International License. Read Full License 


\begin{abstract}
The ATP-binding cassette (ABC) transporter gene family play vital role in substance transportation including heavy metals ion, secondary metabolites and phytohormone across membranous structure. Despite its essential role in crops, still uncovered in potato (Solanum tuberosum L), grown world widely as a 3rd important food crop. Current study identified a total of $54 \mathrm{ABC}$ transporter genes (StABC) in potato genome. The accumulative phylogenetic tree of StABC with Arabidopsis, divided into 8 groups ( $A B C A$ to $A B C H$ ). Among these, $A B C G$ was the largest family covering $90 \%$ of StABC genes followed by ABCB group. The number and architecture of exon-intron varied from gene to gene. However, the sequence alignment and motif analysis demonstrated similar functional and structural sites and motif in all StABC. The syntenic blocks presented segmental duplication among intra and inter genomes. In addition, the presence of stress responsive element in the regulatory regions depicted their role in environmental adaptation. Furthermore, the tissues-specific and stress-specific expression profiling of StABC genes and their validation through Real-Time-qPCR (RT-qPCR) analysis revealed their role in development and stress. The presented results provided useful information for further functional analysis of StABC and can also use as reference study for other important crops.
\end{abstract}

\title{
Introduction
}

The ATP-Binding Cassette (ABC) transporter is one of a largest transporter proteins family, involved in substance transportation across inter and intra cellular membrane. (Jones and George, 2004). In many genome the ABC family of gene is known as a wide family of transporters, distributed into 8 groups, designated $A B C A$ through $A B C H$, although human has only 7 groups (ABCA-ABCG)(Sánchez-Fernández et al., 2001). On the other hand plants don't have $\mathrm{ABCH}$ but they have $\mathrm{ABCl}$ (Lopez-Ortiz et al., 2019). These documented proteins normally have two conserved domains, nucleotide binding domain (NBD) and transmembrane domain (TMD). The NBD bind and hydrolyzes ATP for production of energy, needed for TMD to generate pore for substrate translocation across membrane (Sharom et al., 2011; Zhiyi et al., 2015).

In cellular efflux, the ABCG play a vital role in phytohormone transportation and possessed both domains (NBD-TMD)(Borghi et al., 2015). Literature demonstrated that AtABCG25 and AtABCG4O performed have great contribution in the transportation of abscisic acid (ABA) in Arabidopsis (Kang et al., 2010). Similarly, another gene, AtABCG14 is associated with cytokinin pathways (Ko et al., 2014). It was also observed that ABCG member are involved in tissue growth and development. For instance, AtABCG26/ WBC27 and OsABCG15 has significant role in growth of pollen in Arabidopsis (Qin et al., 2013; Quilichini et al., 2010).

A genome-wide analysis is a detailed method for the identification of genes in a specific family. By this method important information including evolutionary pathway, genetic diversity and relationships among genes and proteins can be obtained, which are helpful for future functional analysis. Several Genome-wide studies carried and identified different number of ABC genes in various land plants including Arabidopsis (Sánchez-Fernández et al., 2001) [15], rice (Garcia et al., 2004)[16], maize (Pang et al., 2013)[17], Lotus japonicus (Sugiyama et al., 2006)[18], grapes (Çakır and Kılıçkaya, 2013)[19], pineapple (Chen et al., 2017)[20] and Hevea brasiliensis (Zhiyi et al., 2015)[4]. However, such research is required in potato genome to explore its role in Solanaceae family.

Potato (Solanum tuberosum) is the fourth largest food crop in the world(Zaynab et al., 2017b). Because of its rich corbohydtrate nutrients, high yield and short life cycle, several countries have been using it as staple crop for long time. Different environmental stresses like salinity, alkali, drought and waterlogging, heat and cold are effecting on the elongation of potato roots, potato respiration and photosynthesis, which are compromising the yield and growth of potato (Li et al., 2019b). In addition to abiotic factors, there are several biotic stresses caused by viruses, fungi and nematode (Dai et al., 2007). So, in order to developed potato plant immunity against these biotic and abiotic stresses, scientist are trying to explore stress responsive genes and their implementation in advance molecular biology including genetic engineering, genome editing and marker assisted breeding and selection to develop new resistant varieties. As ABC gene family is one of stress responsive family involved in plant adaptation. Therefore identification, characterization, expression profiling and structural and functional conserved motifs prediction is necessary in potato genome which is fulfilled by current study results. These results are important for functional validation of StABC in potato crop.

\section{Material And Methods}

\section{Identification of $\mathrm{ABC}$ transporter gene in potato genome}

Solanum tuberosum (Potato) genome was downloaded from phytozome (https://phytozome.jgi.doe.gov/pz/portal.html). In order to find ABC genes in potato, we used Arabidopsis ABC transporter as reference protein, retrieved from TIAR (https://www.arabidopsis.org/). The Blastp program was used for sequence similarity analysis. The Pfam (https://Pfam.sanger.ac.uk/) and CDD (https://www.ncbi.nlm.nih.gov/cdd/) databases were included to validate functional domains. The protein statistics including molecular weight $(\mathrm{MW})$ and iso-electric point (PI) were described through an online ProtParam tool located at ExPASy (https://web.expasy.org/protparam/) server (Gasteiger et al. 2005).

Phylogenetic analysis and sub-cellular localization

Page 2/17 
The phylogenetic tree of selected amino acids sequences was constructed with Arabidopsis ABC transporter proteins using MUSCLE program for alignment and MEGA7 for phylogenetic tree with neighbor-joining-method using parameters: Partial deletion $90 \%$, poisson correction and 1000 boot-strap replicate.The Subcellular localization of S.tuborosum ABC transporter gene was projected through CELLO v2.5(https://cello.life.nctu.edu.tw/).

\section{Chromosomal locations, synteny, and selective pressure analysis}

Information regarding to all known StABCs was retrieved from the PGSC website. The comparative synteny analysis for the visualization of genome conservation was executed through circoletto Tool (tools. bat. infspire.org/circoletto/). Duplicated gene coding sequences were arranged with MEGA7 by using the Muscle (codon) method. The synonymous and non-synonymous substitution rate (Ka; No. of nonsynonymous substitutions per nonsynonymous site, Ks; No. of synonymous substitutions per synonymous site) was calculated with KaKs_Calculator 2.0 software through the MYN method. Furthermore, the divergence time " $t=\mathrm{Ks} / 2 r^{\prime \prime}$ was calculated through exchange rate $r=2.6 \times 10^{-9}$ (Li et al., $\left.2019 \mathrm{a}\right)$.

\section{Intron-exon Distribution And Conserved Motif Identification}

The intron-exon distribution was conducted using Gene Structure Display Server (http://gsds.cbi.pku.edu.cn/) (Guo et al., 2007). For the ABC transporter proteins, the conserve motifs were discovered by online MEME server (http://meme-suite.org/) with the given parameters: optimum width range of $>6$ to $<200$, number of motif; 20 . The distribution of motif along amino acid sequence constructed using TB tools (https://github.com/CJ-Chen/TBtools) software.

\section{Prediction of Cis-element in regulatory regions}

In order to find the cis-acting sites in the regulatory regions of StABC genes, we retrieved the promoter sequence (2000 bp upstream of the initiation codon 'ATG') from PGSC server. The cis-regulatory elements were detected using Plant Cis-acting Regulatory DNA Elements (PlantCARE) (http://bioinformatics.psb.ugent.be/webtools/plantcare/html/).

\section{Tissue-specific expression profiling of StABC genes}

The tissue specific expression analysis was conducted by using publicly accessible RNA-seq data.. The collected data was compiled according to highly expressed tissues such as root, stem and leaf. The original Fragments Per Kilobase Million (FPKM) values were used to draw a heatmap with the help of TBtools (Chen et al., 2020)..

\section{Plant material and sample collection}

Potato plant seeds were taken from National Agriculture Research Center (NARC) Islamabad and planted in a glasshouse under optimum conditions at National Institute for Genomics and Advanced Biotechnology (NIGAB), NARC Islamabad. After 25 days of post-germination, leaf, stem, and roots were collected in replicates and stored in liquid nitrogen for RNA extraction.

\section{Extraction Of Rna And Rt-qper}

Total extraction of RNA for leaves, stem and root were performed by using the RNA isolation Kits (Huayueyang, Beijing, China) according to instructions of company. The 1st strand of cDNA was produced by cDNA Kits, ReverTra ${ }^{\circledR}$ Ace.

To observe ABC genes expression level in the three tissues, a qRT-PCR was performed. The amplification of cDNA was performed with SYBR $B$ Green Real time PCR Master Mix (TOYOBO QPK-210, Shanghai, China) by DNA engine Opticon three thermal cycler (Bio-Rad) with $20 \mu \mathrm{L}$ volume. Protocol for amplification have primary step at $95^{\circ} \mathrm{C}$ for one mintue and by following this step fourty cycle of denaturation of 15 second at $95^{\circ} \mathrm{C}$ was performed. The step of annealing was performed for 15 second at $55^{\circ} \mathrm{C}$. The extension step lasts for 15 second at $72^{\circ} \mathrm{C}$. Target gene magnification was checked regularly in every single cycle with the help of SYBR-green fluorescence dye. The amplification of qRT-PCR specificity was regulated by managing a melting-curve-analysis routinely. Analysis of data were performed by $2-\triangle \triangle \mathrm{CT}$ (Livak and Schmittgen, 2001) and the outcomes were explained on the basis of relative level of expression of gene. For a house-keeping genes the glyceraldehyde-3-phosphate dehydrogenase (GAPDH) gene was utilized (Pimentel et al., 2010). For each experiment three replicates were performed. All the sequence of primer is listed under (Table.S1).

\section{Results}

\section{Physico-chemical properties and ABC Transporter gene identification}


In the present study, we have identified a total of 54 proteins with $\mathrm{ABC}$ transporter domains in the genome of $S$. tuborosum. Same genes with different transcripts were not considered. The details of $54 \mathrm{ABC}$ transporter proteins, including genomic location, chromosome number, description, domain organization, molecular weight, protein length and isoelectric point (pl) were listed in (Table 1). The encoded protein have amino acid range from 84 to $1580 \mathrm{aa}, \mathrm{PI}$ range from 5.91 to 9.69 and the molecular weight range from 9261.82 to $178764.6 \mathrm{KDa}$.

\section{Phylogenetic Analysis For Abc Transporter Genes}

For the investigation of evolutionary relationships of the $54 \mathrm{ABC}$ transporters, by using MEGA7.0 software a phylogenetic tree was designed by method of neighbor-joining. The multiple sequence alignment demonstrated important conserved residues in $A B C$ transposter proteins (Fig. 1). The phylogenetic tree was divided into 7 sub-groups with named as $A B C A, A B C B, A B C C, A B C E, A B C F, A B C G$ and $P G P 1$. Of these, $A B C G$ was the largest subgroup. In case of StABCs, all genes were distributed into two sub-groups mainly ABCG and ABCB (Fig. 2).

\section{Sub-cellular localization for StABC proteins}

Sub-cellular localization of 54 StABC genes were predicted with the help of 2 diverse tools and the higher confidence prediction were determined in the Table 1. Many number of ABC transporter proteins in potato were predicted to localized in plasma membrane although two genes; Stabct3 and Stabct20 localized in mitochondria while only one genes; Stabct7 was found in cytoplasm. In addition, Stabct10, Stabct16 and Stabct54 were in nucleus and single gene (Stabct2) was in inner membrane (Table.1). These suggest that these genes may require for the coordinated expression in both nucleus and other organelles.

Intron-exon structure of $\mathrm{ABC}$ transporters in S.tuborosum

The gene structure of StABC genes was examined to define the proteins diversity. The exons number was ranged between 1 to 28 . A total of nine genes possessed one exon while only one genes; Stabct4 gene had 28 exon. Some genes such as Stabct11, Stabct12, Stabct15 and Stabct2 had simpler structure with 1-2 exons. The gene structure was differing only in length and numbers of exons and introns. In addition some genes carried equall number of exons e.g. Stabct23 and Stabct29 had four exons in each gene (Fig. 3).

\section{Conserved motifs analyses}

The proteins structures are directly associated with their function and the conserved motifs have great potential in maintaining the structure and activity of proteins. Therefore, we presented conserved motifs and their patterns in StABCs. A total of ten conserve motifs were evaluated in order to study the architecture of ABC transporter of S.tuberosm. Motif 1st was observed in all genes of S.tuberosm ABC transporter except the two genes Stabct16 and Stabct20 (Fig. 3). Motifs 2nd to 3rd were present in most of the genes. Some motifs were distinctive with respect to 1 or 2 sub-families. Each sub-family member showed some common and some unique motifs. Motif 6 th, and 7 th consist of 50 amino acids residues, and 2nd and 10th motif was comprised of approximately 29 amino acids (Fig. 4).

\section{Chromosomal distribution and Cis-element of StABC genes}

The chromosomal distribution of StABCs demonstrated that, all genes were distributed unequally on all chromosomes. Highest number of genes were localized on Chr5 and Chr6 with 9 genes followed by Chr11 (8 genes), Chr8 and Chr12 (7 genes), Chr1 (5 genes,), Chr3,4,7,8 and Chr9 (4 genes ) and Chr2 (1 gene)(Table.1). On the basis of significance of cis-element in promoters region on gene regulation the identified cis-elements were categories into different groups including stress responses, development and phytohormone response. Forinstqance, the most common cisactingf site was MeJRE and anaerobic induction. Followed by ABRE, GR and SARE (Fig. 5).

\section{Gene duplications of StABC}

The value of $\mathrm{Ka} / \mathrm{Ks}$ for each duplicated gene pair was estimated to calculate the molecular evolution rate. The Ka/Ks $>1$ considered as + ve selection effect, while $\mathrm{Ka} / \mathrm{Ks}<1$ as purifying selection and $\mathrm{Ka} / \mathrm{Ks}=1$ with neutral selection among the duplicated genes (Yang and Bielawski, 2000). The cosmic majority of StABC genes were presented the Ks value greater than 0.52 , while the corresponding deviation time can be more than 100 million years ago (MYA). Fascinatingly, the Ks value of the duplicated pair genes (Stabct30/Stabct52) was 2.94, while the corresponding duplication time may be 93.98 million years ago (MYA) (Table 2).

\section{Comparative synteny of StABCs}

Comparative synteny analysis among Arabidopsis (Arabidopsis thaliana), potato (S.tuberosum), and tomato (Solanum lycoperiscum) demonstrated a remarkable relationship regarding gene evolution, expansion, duplication, triplication, and function. It was noticed that the tomato Solyc05g053570.2 gene sequence illustrated synteny among potato gene sequence Stabct48. Similarly, potato gene sequence Stabct19 illustrated synteny with Solyc12g100190.1 (Fig. 6).

Tissue-specific expression profiling 
The tissues specific expression profiling results suggested that different tissues had different number of differential genes. For instance, twenty one $A B C$ transporter genes showed relatively high transcriptional abundance in leaves. However, some genes did not show any expression, whereas others showed tissue-specific expression. For instance, Stabct17, Stabc18, Stabc6, Stabc19 showed high transcript level in leaf, root and root, respectively. Some genes, expressed in more than one tissues like, Stabc21 and Stabc50 had similar expression in root and stem. Most of the gene did not show any expression (Figure. 7A).

\section{Stress-specific Expression Profiling Of Stabcs}

In order to find the putative role of StABCs under different abiotic stresses we have also analysis the RNA-seq data of StABCs under heat, Indole acetic acid (IAA), abscisic acid (ABA) and P. infestant stresses. A total of twenty nine StABC genes showed expressed under heat stress. The expression of some genes enhanced under heat stress i.e. Stabc3, Stabc10, Stabc36, Stabc51, and Stabc41. Similarly, some genes had remarkable expression under $P$. infestation while some had in ABA and IAA. (Fig. 7B)

RT-qPCR Analysis

The RT-qPCR analysis also validated the expression analysis of StABC6, StABC17, StABC18,StABC21, and StABC50 genes in roots, stems, and leaves. The relative expression pattern demonstrated that StABC6 and StABC18 genes had a higher expression in the roots compared to stems and leaves. The genes StABC17,StABC21, and StABC50 had higher expression in the leaf (Fig. 8).

\section{Discussion}

Potatoes are an important component of the world's food supply (Zaynab et al., 2017b). During their growth and developmental process, potatoes are subjected to a variety of stresses (Quandahor et al., 2021). In order to develops plant immunity, several stress responsive genes were identified. Of these $A B C$ transporter is one of an important genes family that are involved in various regulatory processes such as growth and development and response to various biotic and abiotic stresses(Rehman et al., 2020). In current study we have identified a total of 54 StABC genes in potato genome which is quite similar to other crops like one hundred thirty fıve member in Vitis vinifera(Çakır and Kılıçkaya, 2013) (Çakır and Kılıçkaya, 2013), one hundred thirty five member in maize (Pang et al., 2013)(Pang et al., 2013), one hundred twenty one member in rice (Garcia et al., 2004)(Garcia et al., 2004), one hundred thirty one member in Arabidopsis thaliana (Sánchez-Fernández et al., 2001).

The phylogenetic anlaysis demonstrated that most of the identified StABCs were belong to ABCG groups followed by ABCB group, showing their abundance in potato genome. The accomulaitve phylogenetic tree divided into 8 groups (ABCA-ABCl). Literature study demonstrated that AtABCB19 and AtABCB1 in arabidoposis have a strong role in post-embryonic organs and cytoplasmic flow with a synergistic role[52,53]. Similarly, the AtABCG11 is involved in the transportation of grease substance and paraffin wax and found in leaf, stem and floral organ. So, the StABCB and ABCG may have similar role in potato genome.

The structural analysis of StABC proteins will be helpful in functional analysis. The evolutionary tree revealed that intron-exon arrangements had shaped the evolution of the gene family (Flagel and Wendel, 2009; Moore and Purugganan, 2005). It corresponds to earlier scientific findings that some genes tend to retain in plants that may have short introns or may not have introns during evolution (Mattick and Gagen, 2001). The expression levels of genes with no and few introns are low in plant genomes (Mattick and Gagen, 2001). Furthermore, compact gene structure may allow rapid expression response to exogenous or endogenous stimulus (Jeffares et al., 2008). Our gene structure analysis results also suggested that StABC gene sequences presented the same number of exons/introns with similar functional features because they may have originated during evolutionary processes in the course of duplication events (Bari et al., 2018; Waqas et al., 2019).

Cis-elements studies can present an important foundation for additional functional dissection of StABC genes. We found that all StABC promoters contain one or more stress-responsive cis-elements such as LTR (low-temperature response element), TATC- box, and P-box (MeJA-response element), ABRE (ABA response element), and SARE (SA response element). These Cis-elements play a vital role in stress response by regulating stress-responsive genes (Wu et al., 2014). Therefore, these critical cis-acting sites in the StABCs suggested their response under various environmental stresses.

The genome size, gene distribution, and duplications in the genome are the main factors of genetic diversity among land plants. The genetic duplication character has long been identified during the origins of gene families' evolutionary novelty, expression, and complexity. We also found some duplication events in $S t A B C$, which may perform a vital role in $S t A B C$ amplification. Because gene duplication is an essential feature in the neofunctionalization, expansion, and diversification of gene family (Lavin et al., 2005). Similarly, the distribution and mapping of StABC genes at the chromosome level will help potato breeders to develop new varieties with the desired traits. These mapping can be used in marker assisted breading and selection of desired offspring.

The expression profiling of genes in different tissues improve our understanding of the tissue-specific and dynamic expression of the $A B C$ genes in potato. Some StABC transporter (Stabct) were expressed uniquely in vegetative organs, Stabct6,Stabct17, and Stabct18 in the roots and leaves, 
indicating their potential role in the vegetative tissue development in potato. The current study reported higher transcripts abundance of Stabcts in the leaves and such evidence was also observed in previous studies (Shi et al., 2020) and supported our results. The upregulation of Stabct 17 in leaves tissues also validated with RT-qPCR analysis, which was presented similar result. It suggests that potato ABC transporter genes have a substantial role in plant growth, development and response to various stresses.

To date, an increasing number of studies have shown that members of the ABC transporters family can play an important role in response to different stresses (Wang et al., 2021). In potato we observed upregulation of Stabct3 and Stabct51 under heat stress and similar results also seen in rice (Moon and Jung, 2014). Hormones can affect the physiological and biochemical reactions of plants through a variety of signal transduction pathways (Fatima et al., 2021; Zaynab et al., 2017a). IAA and ABA are important hormones in the plant immune system. A number of studies have revealed that $A B C$ s are not only involved in stress response but also in developmental and hormonal signaling (Kumar et al., 2020).. Under ABA and IAA treatment, 26 and 24 Stabct genes upregulated, respectively. The upregulation of these genes might have putative role in potato to regulater abiotic stresses (El Guizani et al., 2014). The expression and their clustering also highlighted a strong correlation of genesc clusters and their expression under different tissues and stresses. These co-expression and co-occurrence shows their essential role in plant adaptation under divers' environmental stresses.

The $P$. infenstans pathogenic stress expression profiling of Stabct also demonstrated up regulation of two important genes e.g. Stabct48, and Stabct49. Similar results were also observed in Oryza sativa (rice) ABC gene, which was transcriptionally upregulated upon blast fungus (Pyricularia grisea) infection. (Xiong et al., 2001). Sevral functional studies of ABCG gene showed their role under biotic stress in potato (Ruocco et al., 2011). Most interestingly, infection of potato plants by $P$. infenstans led to the tremendous up regulation of StPDR1 and StPDR2 expression in leaves at $48 \mathrm{hrs}$ post infection.

\section{Conclusion}

Taken together, a total of $54 \mathrm{ABC}$ transporter genes were reported in potatoes. The comparative evolutionary analysis concluded that, the $\mathrm{ABCG}$ group was the largest $A B C$ transporter group in potato. The conserved structural and functional motifs associated to $A B C$ transporter domain were present in all Stabcts with slight variation among groups and members. The tissue-specific and stress-specific expression profiling of Stabcts demonstrated their putative role in potato developmental stages and environmental adaptation. These results are essential steps for potato growth and development under various environemtnal stresses.

\section{References}

Bari A, Farooq M, Hussain A, Muhammad T, Abbas M, Mustafa G, Karim A, Ahmed I, Hussain T (2018) Genome-wide bioinformatics analysis of aquaporin gene family in maize (Zea mays L.). Journal of Phylogenetics Evolutionary Biology 6:197

Borghi L, Kang J, Ko D, Lee Y, Martinoia E (2015) The role of ABCG-type ABC transporters in phytohormone transport. Biochem Soc Trans 43:924930

Çakır B, Kılıçkaya O (2013) Whole-genome survey of the putative ATP-binding cassette transporter family genes in Vitis vinifera. PloS one 8:e78860

Chen C, Chen H, Zhang Y, Thomas HR, Frank MH, He Y, Xia R (2020) TBtools: an integrative toolkit developed for interactive analyses of big biological data. Molecular plant 13:1194-1202

Chen P, Li Y, Zhao L, Hou Z, Yan M, Hu B, Liu Y, Azam SM, Zhang Z, Liu L (2017) Genome-wide identification and expression profiling of ATPbinding cassette $(A B C)$ transporter gene family in pineapple (Ananas comosus (L.) Merr.) reveal the role of AcABCG38 in pollen development. Frontiers in plant science 8:2150

Dai LY, Liu XL, Xiao YH, Wang GL (2007) Recent advances in cloning and characterization of disease resistance genes in rice. J Integr Plant Biol 49:112-119

El Guizani T, Blanc N, Triki S, St-Pierre B, Ducos E (2014) Expression pattern of AtABCC13/MRP11 reveals developmental, hormonal, and nutritional regulations. Biologia plantarum 58:231-240

Fatima M, Ma X, Zhou P, Zaynab M, Ming R (2021) Auxin regulated metabolic changes underlying sepal retention and development after pollination in spinach. BMC plant biology 21:1-15

Flagel LE, Wendel JF (2009) Gene duplication and evolutionary novelty in plants. New Phytol 183:557-564

Garcia O, Bouige P, Forestier C, Dassa E (2004) Inventory and comparative analysis of rice and Arabidopsis ATP-binding cassette (ABC) systems. Journal of molecular biology 343:249-265 
Hwang J-U, Song W-Y, Hong D, Ko D, Yamaoka Y, Jang S, Yim S, Lee E, Khare D, Kim K (2016) Plant ABC transporters enable many unique aspects of a terrestrial plant's lifestyle. Molecular plant 9:338-355

Jeffares DC, Penkett CJ, Bähler J (2008) Rapidly regulated genes are intron poor. Trends in genetics 24:375-378

Jones P, George A (2004) The ABC transporter structure and mechanism: perspectives on recent research. Cellular Molecular Life Sciences CMLS 61:682-699

Kang J, Hwang J-U, Lee M, Kim Y-Y, Assmann SM, Martinoia E, Lee Y (2010) PDR-type ABC transporter mediates cellular uptake of the phytohormone abscisic acid. Proceedings of the National Academy of sciences 107, 2355-2360

Ko D, Kang J, Kiba T, Park J, Kojima M, Do J, Kim KY, Kwon M, Endler A, Song W-Y (2014) Arabidopsis ABCG14 is essential for the root-to-shoot translocation of cytokinin. Proceedings of the National Academy of sciences 111, 7150-7155

Kumar K, Raina SK, Sultan SM (2020) Arabidopsis MAPK signaling pathways and their cross talks in abiotic stress response. Journal of Plant Biochemistry and Biotechnology, 1-15

Lavin M, Herendeen PS, Wojciechowski MF (2005) Evolutionary rates analysis of Leguminosae implicates a rapid diversification of lineages during the tertiary. Syst Biol 54:575-594

Li G, Hou M, Liu Y, Pei Y, Ye M, Zhou Y, Huang C, Zhao Y, Ma H (2019a) Genome-wide identification, characterization and expression analysis of the non-specific lipid transfer proteins in potato. BMC Genomics 20:1-14

Li Y, He H, He L-F (2019b) Genome-wide analysis of the MATE gene family in potato. Molecular biology reports 46:403-414

Lopez-Ortiz C, Dutta SK, Natarajan P, Peña-Garcia Y, Abburi V, Saminathan T, Nimmakayala P, Reddy UK (2019) Genome-wide identification and gene expression pattern of ABC transporter gene family in Capsicum spp. PloS one 14:e0215901

Mattick JS, Gagen MJ (2001) The evolution of controlled multitasked gene networks: the role of introns and other noncoding RNAs in the development of complex organisms. Molecular biology evolution 18:1611-1630

Moon S, Jung K-H (2014) Genome-wide expression analysis of rice ABC transporter family across spatio-temporal samples and in response to abiotic stresses. Journal of plant physiology 171:1276-1288

Moore RC, Purugganan MD (2005) The evolutionary dynamics of plant duplicate genes. Curr Opin Plant Biol 8:122-128

Pang K, Li Y, Liu M, Meng Z, Yu Y (2013) Inventory and general analysis of the ATP-binding cassette (ABC) gene superfamily in maize (Zea mays L.). Gene 526:411-428

Qin P, Tu B, Wang Y, Deng L, Quilichini TD, Li T, Wang H, Ma B, Li S (2013) ABCG15 encodes an ABC transporter protein, and is essential for postmeiotic anther and pollen exine development in rice. Plant Cell Physiol 54:138-154

Quandahor P, Gou Y, Lin C, Coulter JA, Liu C (2021) Comparison of root tolerance to drought and aphid (Myzus persicae Sulzer) resistance among different potato (Solanum tuberosum L.) cultivars. Scientific reports 11:1-13

Quilichini TD, Friedmann MC, Samuels AL, Douglas CJ (2010) ATP-binding cassette transporter G26 is required for male fertility and pollen exine formation in Arabidopsis. Plant Physiol 154:678-690

Rehman N, Khan MR, Abbas Z, Rafique RS, Zaynab M, Qasim M, Noor S, Inam S, Ali GM (2020) Functional characterization of mitogen-activated protein kinase kinase (MAPKK) gene in halophytic Salicornia europaea against salt stress. Environ Exp Bot 171:103934

Ruocco M, Ambrosino P, Lanzuise S, Woo SL, Lorito M, Scala F (2011) Four potato (Solanum tuberosum) ABCG transporters and their expression in response to abiotic factors and Phytophthora infestans infection. Journal of plant physiology 168:2225-2233

Sánchez-Fernández Ro, Davies TE, Coleman JO, Rea PA (2001) The Arabidopsis thaliana ABC protein superfamily, a complete inventory. J Biol Chem 276:30231-30244

Sharom FJ, Kretzschmar T, Burla B, Lee Y, Martinoia E, Nagy R (2011) Functions of ABC transporters in plants. Essays Biochem 50:145-160

Shi M, Wang S, Zhang Y, Wang S, Zhao J, Feng H, Sun P, Fang C, Xie X (2020) Genome-wide characterization and expression analysis of ATPbinding cassette $(A B C)$ transporters in strawberry reveal the role of FvABCC11 in cadmium tolerance. Sci Hortic 271:109464 
Sugiyama A, Shitan N, Sato S, Nakamura Y, Tabata S, Yazaki K (2006) Genome-wide analysis of ATP-binding cassette (ABC) proteins in a model legume plant, Lotus japonicus: comparison with Arabidopsis ABC protein family. DNA research 13:205-228

Theodoulou FL, Kerr ID (2015) ABC transporter research: going strong 40 years on. Biochem Soc Trans 43:1033-1040

Wang Z, Wan Y, Meng X, Zhang X, Yao M, Miu W, Zhu D, Yuan D, Lu K, Li J (2021) Genome-Wide Identification and Analysis of MKK and MAPK Gene Families in Brassica Species and Response to Stress in Brassica napus. Int J Mol Sci 22:544

Waqas M, Azhar MT, Rana IA, Azeem F, Ali MA, Nawaz MA, Chung G, Atif RM (2019) Genome-wide identification and expression analyses of WRKY transcription factor family members from chickpea (Cicer arietinum L.) reveal their role in abiotic stress-responses. Genes genomics 41:467-481

Wu J, Wang J, Pan C, Guan X, Wang Y, Liu S, He Y, Chen J, Chen L, Lu G (2014) Genome-wide identification of MAPKK and MAPKKK gene families in tomato and transcriptional profiling analysis during development and stress response. PloS one 9:e103032

Xiong L, Lee M-W, Qi M, Yang Y (2001) Identification of defense-related rice genes by suppression subtractive hybridization and differential screening. Mol Plant Microbe Interact 14:685-692

Yang Z, Bielawski JP (2000) Statistical methods for detecting molecular adaptation. Trends Ecol Evol 15:496-503

Zaynab M, Kanwal S, Furqan M, Islam W, Noman A, Ali GM, Rehman N, Zafar S, Sughra K, Jahanzab M (2017a) Proteomic approach to address low seed germination in Cyclobalnopsis gilva. Biotechnol Lett 39:1441-1451

Zaynab M, Kanwal S, Hussain I, Qasim M, Noman A, Iqbal U, Ali GM, Bahadar K, Jamil A, Sughra K (2017b) Rice chitinase gene expression in genetically engineered potato confers resistance against Fusarium solani and Rhizictonia solani. PSM Microbiology 2:63-73

Zhiyi N, Guijuan K, Yu L, Longjun D, Rizhong Z (2015) Whole-transcriptome survey of the putative ATP-binding cassette (ABC) transporter family genes in the latex-producing laticifers of Hevea brasiliensis. PLoS One 10:e0116857

\section{Tables}

Table 1 List of identified putative Stabcts and their features. CDS; coding sequence, pl; iso-electric point 


\begin{tabular}{|c|c|c|c|c|c|c|c|c|c|}
\hline Gene & Location Start -end & $\begin{array}{l}\text { Genomics } \\
\text { sequence }\end{array}$ & CDS & Peptide & Chromosomes & $\begin{array}{l}\text { Number } \\
\text { of } \\
\text { amino } \\
\text { acids }\end{array}$ & $\begin{array}{l}\text { Molecular } \\
\text { weight }\end{array}$ & pl: & $\begin{array}{l}\text { Sub-cellular } \\
\text { localization }\end{array}$ \\
\hline Stabct1 & $58762883 . .58769064$ & 6182 & 2271 & 756 & 4 & 756 & 84481.71 & 8.89 & PlasmaMembrane \\
\hline Stabct2 & $11327196 . .11336370$ & 9175 & 1812 & 603 & 11 & 603 & 66691.78 & 9.08 & InnerMembrane \\
\hline Stabct3 & $47266008 . .47267889$ & 1882 & 1317 & 438 & 8 & 438 & 48277.62 & 8.98 & Mitochondrial \\
\hline Stabct4 & $41606331 . .41613682$ & 7352 & 4743 & 1580 & 8 & 1580 & 178764.6 & 6.12 & PlasmaMembrane \\
\hline Stabct5 & $41628506 . .41631135$ & 2630 & 1878 & 625 & 8 & 625 & 71347.73 & 7.06 & PlasmaMembrane \\
\hline Stabct6 & $60412413 . .60421201$ & 8789 & 4503 & 1500 & 3 & 1500 & 170088.85 & 8.24 & PlasmaMembrane \\
\hline Stabct7 & $59678366 . .59679025$ & 660 & 504 & 167 & 12 & 167 & 17798.29 & 5.93 & Cytoplasmic \\
\hline Stabct8 & $59696990 . .59702667$ & 5678 & 3864 & 1287 & 12 & 1287 & 139652.46 & 6.74 & PlasmaMembrane \\
\hline Stabct9 & $40225813 . .40235522$ & 9710 & 3864 & 1287 & 11 & 1287 & 141384.58 & 9.2 & PlasmaMembrane \\
\hline Stabct10 & $641972 . .644313$ & 2243 & 1209 & 402 & 4 & 402 & 44884.16 & 8.9 & Nuclear \\
\hline Stabct11 & $6566287 . .6568374$ & 2088 & 2088 & 695 & 4 & 695 & 77892.19 & 9.03 & PlasmaMembrane \\
\hline Stabct12 & $6560427 . .6562601$ & 2175 & 2175 & 724 & 4 & 724 & 81057.78 & 9.27 & PlasmaMembrane \\
\hline Stabct13 & $55507940 . .555111125$ & 3186 & 1081 & 359 & 6 & 359 & 39889.86 & 9.25 & PlasmaMembrane \\
\hline Stabct14 & $55574774 . .55583712$ & 8939 & 3219 & 1072 & 6 & 1072 & 118757.27 & 8.83 & PlasmaMembrane \\
\hline Stabct15 & $52773860 . .52775722$ & 1863 & 1863 & 620 & 7 & 620 & 69167.78 & 8.78 & PlasmaMembrane \\
\hline Stabct16 & $82110892 . .82111440$ & 549 & 339 & 112 & 1 & 112 & 12193.81 & 9.69 & Nuclear \\
\hline Stabct17 & $82091452 . .82095286$ & 3835 & 1890 & 629 & 1 & 629 & 69765.02 & 7.53 & PlasmaMembrane \\
\hline Stabct18 & $49017339 . .49024379$ & 7041 & 4284 & 1427 & 5 & 1427 & 161056.36 & 7.02 & PlasmaMembrane \\
\hline Stabct19 & $48998602 . .49005673$ & 7072 & 3738 & 1245 & 5 & 1245 & 140318.44 & 7.99 & PlasmaMembrane \\
\hline Stabct20 & $48992723 . .48993463$ & 741 & 267 & 88 & 5 & 88 & 9744.33 & 9.25 & Mitochondrial \\
\hline Stabct21 & $50423518 . .50427078$ & 3561 & 2124 & 707 & 3 & 707 & 79063.15 & 8.6 & PlasmaMembrane \\
\hline Stabct22 & $813841 . .815847$ & 2007 & 1671 & 556 & 12 & 556 & 62046.39 & 8.97 & PlasmaMembrane \\
\hline Stabct23 & $808495 . .811827$ & 3333 & 1890 & 629 & 12 & 629 & 69913.27 & 8.47 & PlasmaMembrane \\
\hline Stabct24 & $2753615 . .2761615$ & 8061 & 1938 & 645 & 11 & 645 & 72455.58 & 8.59 & PlasmaMembrane \\
\hline Stabct25 & $46442609 . .46446926$ & 4318 & 1959 & 652 & 5 & 652 & 72463.08 & 7.95 & PlasmaMembrane \\
\hline Stabct26 & 78728173...78733344 & 5172 & 2673 & 890 & 1 & 890 & 100244.39 & 7.16 & PlasmaMembrane \\
\hline Stabct27 & $3599414 . .3601252$ & 1839 & 1384 & 462 & 8 & 462 & 52051.61 & 8.85 & PlasmaMembrane \\
\hline Stabct28 & $23010110 . .23018202$ & 8093 & 3654 & 1217 & 12 & 1217 & 137720.46 & 8.52 & PlasmaMembrane \\
\hline Stabct29 & $53551639 . .53554802$ & 3164 & 1842 & 613 & 7 & 613 & 68483.95 & 8.55 & PlasmaMembrane \\
\hline Stabct30 & $75147184 . .75150158$ & 2975 & 2499 & 832 & 1 & 832 & 93962.11 & 8.91 & PlasmaMembrane \\
\hline Stabct31 & $5021794 . .5029583$ & 7790 & 4341 & 1446 & 11 & 1446 & 164225.77 & 6.81 & PlasmaMembrane \\
\hline Stabct32 & $5013377 . .5020180$ & 6804 & 4179 & 1392 & 11 & 1392 & 158626.74 & 7.53 & PlasmaMembrane \\
\hline Stabct33 & $41997222 . .42005704$ & 8483 & 2172 & 723 & 11 & 723 & 80509.68 & 8.86 & PlasmaMembrane \\
\hline Stabct34 & $50049590 . .50051416$ & 1827 & 1827 & 608 & 7 & 608 & 68618.94 & 8.76 & PlasmaMembrane \\
\hline Stabct35 & $44358550 . .44360340$ & 1791 & 1791 & 596 & 3 & 596 & 66398.86 & 9.31 & PlasmaMembrane \\
\hline Stabct36 & $2557631 . .2565744$ & 8114 & 2181 & 726 & 1 & 726 & 80223.77 & 8.84 & PlasmaMembrane \\
\hline Stabct37 & $55781265 . .55790061$ & 8797 & 3387 & 1128 & 7 & 1128 & 125437.86 & 8.27 & PlasmaMembrane \\
\hline Stabct38 & $51897691 . .51899616$ & 1926 & 1926 & 641 & 5 & 641 & 72186.33 & 9.09 & PlasmaMembrane \\
\hline
\end{tabular}




\begin{tabular}{|llllllllll|} 
Stabct39 & $51014601 . .51019460$ & 4860 & 3048 & 1015 & 5 & 1015 & 114928.23 & 8.36 & PlasmaMembrane \\
\hline Stabct40 & $51000420 . .51006217$ & 5798 & 3432 & 1143 & 5 & 1143 & 129243.28 & 6.8 & PlasmaMembrane \\
\hline Stabct41 & $50607365 . .50609636$ & 2272 & 2175 & 724 & 5 & 724 & 80435.99 & 8.98 & PlasmaMembrane \\
\hline Stabct42 & $60789857 . .60796562$ & 6706 & 4308 & 1435 & 12 & 1435 & 162634.28 & 8.55 & PlasmaMembrane \\
\hline Stabct43 & $7152699 . .7154924$ & 2226 & 2226 & 741 & 9 & 741 & 82584.39 & 9.3 & PlasmaMembrane \\
\hline Stabct44 & $53683922 . .53685907$ & 1986 & 1986 & 661 & 3 & 661 & 74141.98 & 8.55 & PlasmaMembrane \\
\hline Stabct45 & $46111847 . .46120421$ & 8575 & 4224 & 1407 & 6 & 1407 & 159992.74 & 8.78 & PlasmaMembrane \\
\hline Stabct46 & $39864565 . .39873867$ & 9303 & 4011 & 1336 & 11 & 1336 & 151501.91 & 6.28 & PlasmaMembrane \\
\hline Stabct47 & $52893988 . .52897879$ & 3892 & 1971 & 656 & 6 & 656 & 73689.67 & 7.6 & PlasmaMembrane \\
\hline Stabct48 & $58529046 . .58537413$ & 8368 & 4080 & 1359 & 9 & 1359 & 153519.13 & 5.91 & PlasmaMembrane \\
\hline Stabct49 & $58544464 . .58551480$ & 7017 & 4302 & 1433 & 9 & 1433 & 161618.19 & 6.02 & PlasmaMembrane \\
\hline Stabct50 & $37030656 . .37036265$ & 5610 & 1359 & 452 & 11 & 452 & 49897.56 & 7.68 & PlasmaMembrane \\
\hline Stabct51 & $3945546 . .3955866$ & 10321 & 2133 & 710 & 5 & 710 & 78004.55 & 7.03 & PlasmaMembrane \\
\hline Stabct52 & $61225165 . .61227965$ & 2801 & 2190 & 729 & 9 & 729 & 81481.54 & 9.12 & PlasmaMembrane \\
\hline Stabct53 & $51148426 . .51155060$ & 6635 & 3438 & 1145 & 12 & 1145 & 130281.63 & 6.92 & PlasmaMembrane \\
\hline Stabct54 & $14392117 . .14392497$ & 381 & 255 & 84 & 2 & 84 & 9261.82 & 7.61 & Nuclear \\
\hline
\end{tabular}

Table 2. Gene duplication and selection pressure of Stabcts. Ka; No. of nonsynonymous substitutions per nonsynonymous site, Ks; No. of synonymous substitutions per synonymous site, MYA; Million years ago

\begin{tabular}{|llllll|}
\hline Seq_1 & Seq_2 & Ka & Ks & Ka_Ks & Time(MYA) \\
\hline Stabct21 & Stabct47 & 0.112387323 & 0.855380415 & 0.131388703 & 21.61294671 \\
\hline Stabct13 & Stabct33 & 0.085755124 & 0.418796211 & 0.204765758 & 16.49136991 \\
\hline Stabct16 & Stabct17 & 0.060577816 & 0.394385524 & 0.153600506 & 11.64958002 \\
\hline Stabct24 & Stabct25 & 0.189904664 & 1.059093651 & 0.179308661 & 36.52012767 \\
\hline Stabct8 & Stabct9 & 0.382823768 & 2.245388104 & 0.170493362 & 73.61995534 \\
\hline Stabct15 & Stabct34 & 0.303168999 & 1.309777872 & 0.231465965 & 58.30173062 \\
\hline Stabct30 & Stabct52 & 0.48869732 & 2.942385034 & 0.166088841 & 93.98025378 \\
\hline Stabct11 & Stabct12 & 0.056643031 & 0.253656609 & 0.223305956 & 10.89289067 \\
\hline Stabct37 & Stabct54 & 0.142248336 & 0.427450302 & 0.332783333 & 27.35544926 \\
\hline Stabct3 & Stabct27 & 0.086200038 & 1.020628207 & 0.084457825 & 16.57693046 \\
\hline Stabct22 & Stabct23 & 0.090579633 & 0.288029867 & 0.314480001 & 17.41916017 \\
\hline Stabct31 & Stabct40 & 0.099321903 & 0.58069016 & 0.171041133 & 19.10036598 \\
\hline
\end{tabular}

\section{Figures}




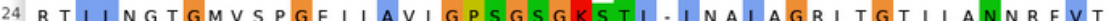

AT3G13220
Stabct37

Stabct10

AT 3625620

AT1G31770

Stabct3

AT4G27420

$81 R T$ I $R$ (

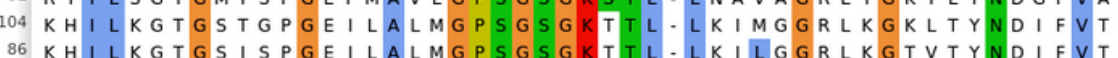

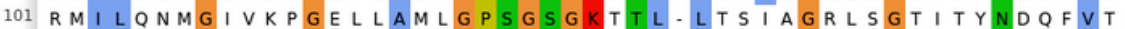

$97 R L V L K C S G$ I VKPGELLAMLGP S G S G K T T L - VT A LAGRLSGTVS Y NGEFVT.

79 K T I LNG TGMVCPGEFLAMLGPSGS G K T T L. LSA LGGRLSGKVMYNGQFVA.

72 K T I LNG TG I V C P GEMLAMLGPS G G K T T L. LTALGGRLSGKI TYNSQFVA.

64 R T I LKG TG I V K P GE I LAMLGPSGS K T S L. LTALGGRVTGN I S Y N N K FV T

Stabct29

Stabct22

Stabct23

Stabct38

AT2G13610

Stabct34

Stabct15

51 K I I LRG TG I VFPGEMLAMLGPSGSG K T T L. L T G LGGRLEGT I T Y NGMFVT

1
59

57 RHVLKDNCRAKTGE I LA I VGPSGAGKSTF. LEVLAGKISGSIFVNQKYVT

60 KHVLKGTCRAKPWE I LA IVGP S GA GKS SL. LE I LAARLTGSVYVNKRYVT.

47 T Y I LRNSCEAKPGE I TAVVGPSGAGKTTL. LD I LAGNVSGHVLVNDQYVT.

$49 K F$ I I KDNCEARPGE I TA I A GPSGAGKTTL-LE I LAGKITGEVLVNGHYVT

AT1G53270

Stabct35

AT 4 G25750

AT5G52860

48 KV I LKDSCDARSAE I TA I A G P S G A G K T T L. LE I LAGKVSGQVLVNGRFVP

46 I N I LKDSFTAYPSQ I LA I VGPSGAG KS T L. LD I LAAGTSGTLLLNSLYVP.

$30 .-$ I LRN TLTSHPS Q I LA I I GPS GAGKSTL. LD I LAARTSGS I L L NSVYVP.

42 S F I LR N T L T A P TE I L AVVG P S G A K KS T L. LD I LASKTSGS I L LNS I Y V P.

Stabct44

92 T T I LKS SFVAGCSE I LA I VG P S G T G

ATSG19410

\section{Figure 1}

multiple sequence alignment of $A t A B C$ and $S t A B C$

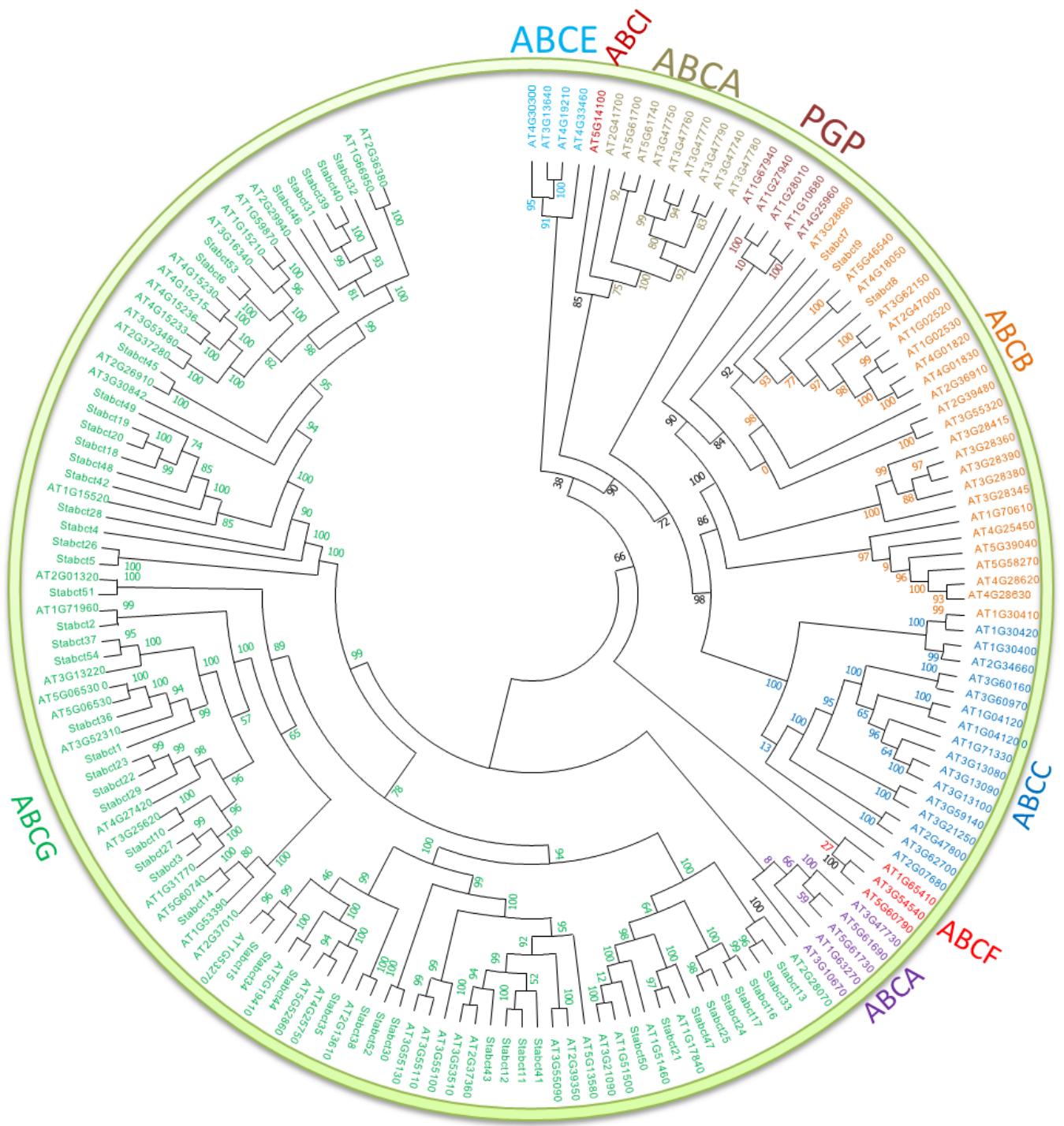

Page $11 / 17$ 


\section{Figure 2}

An accumulative phylogenetic tree containing Arabidopsis $A B C$ and Potato $A B C$ proteins. Numbers on the nodes are representing bootstrap values.

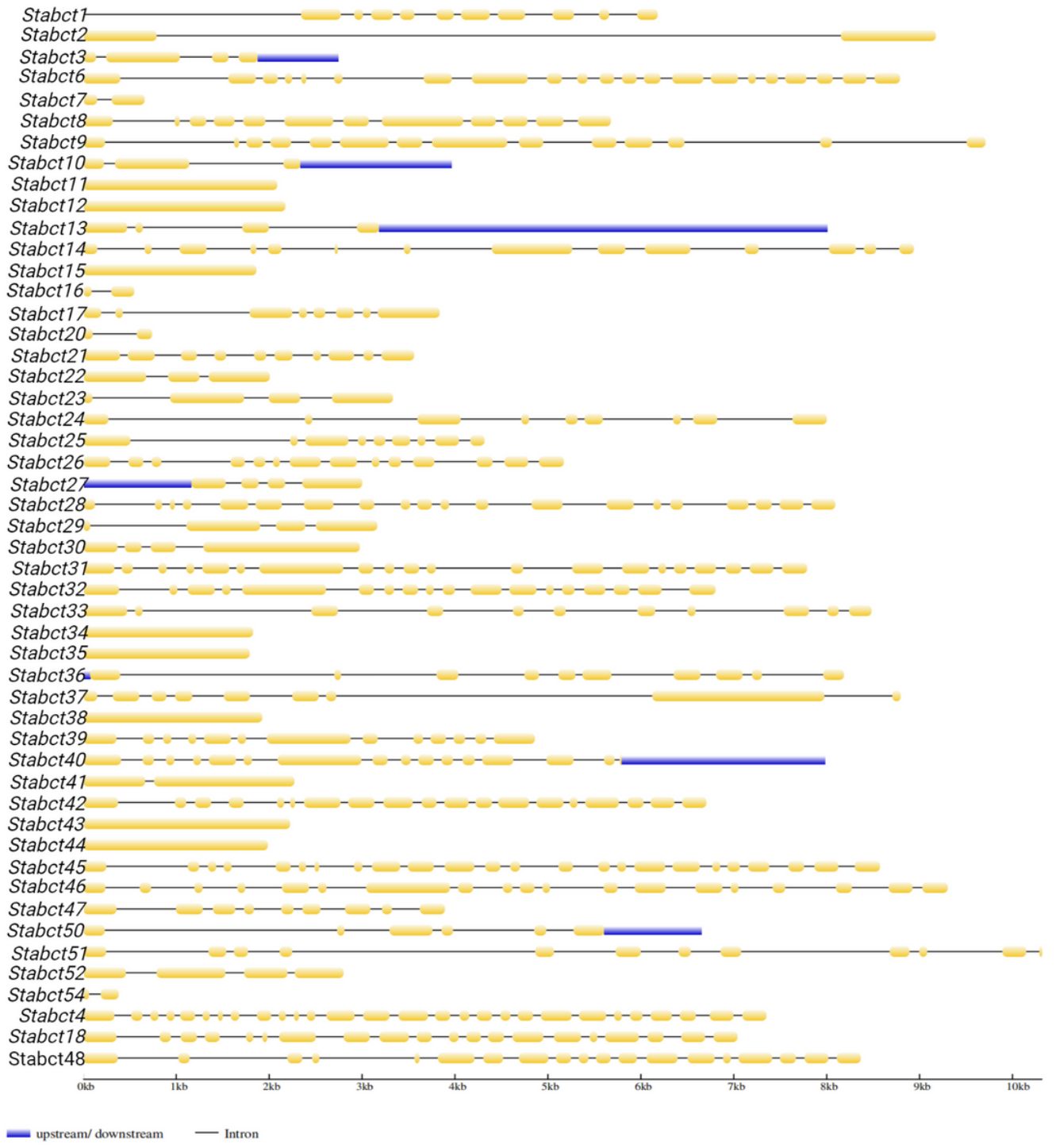

Legend:

CDS upstream/downstream - Intron

\section{Figure 3}

Distribution of exons, introns and UTR (untranslated regions) in Stabct genes sequence. 


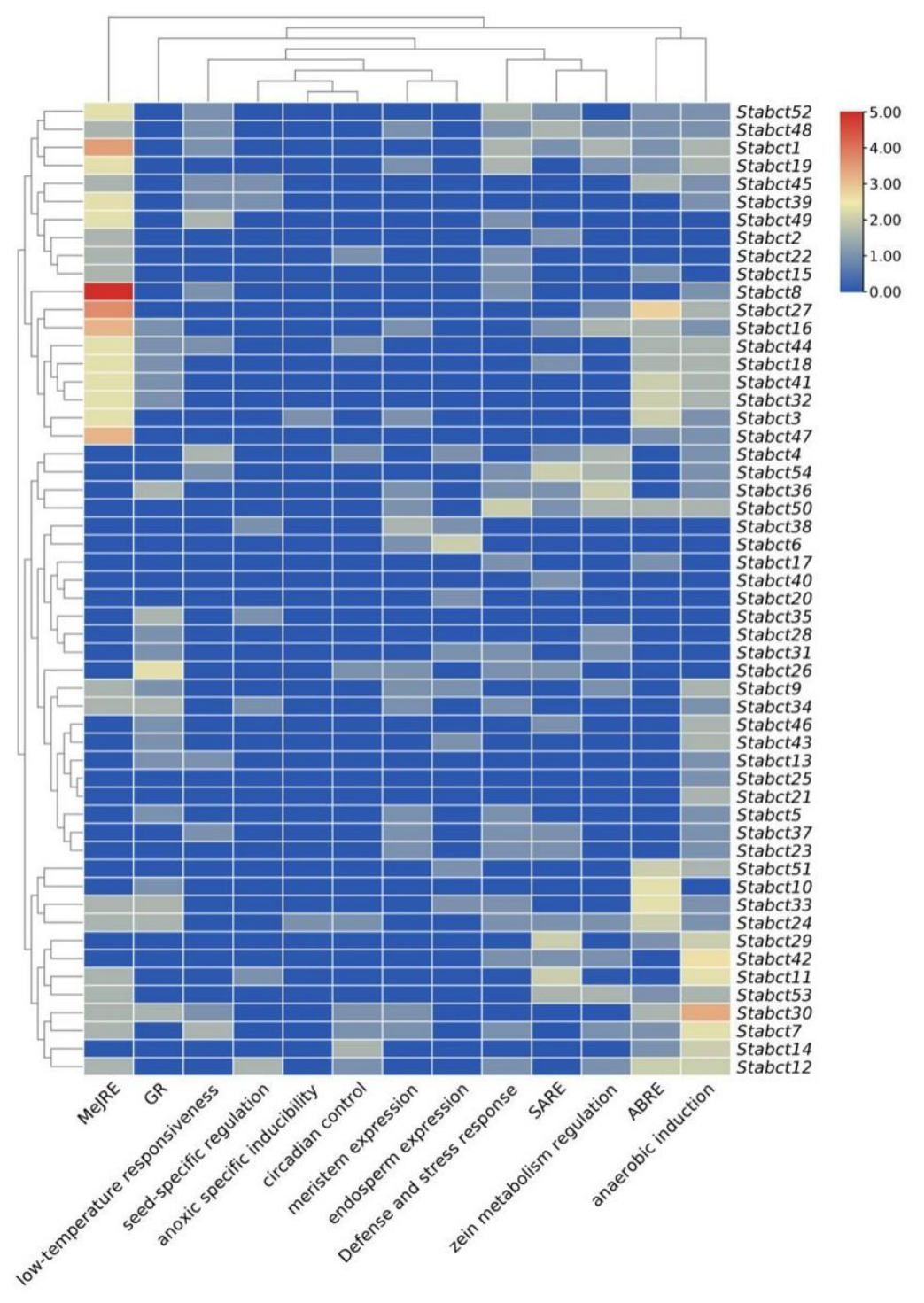

Figure 4

De-novo MEME motifs distributions in the Stabcts proteins. 


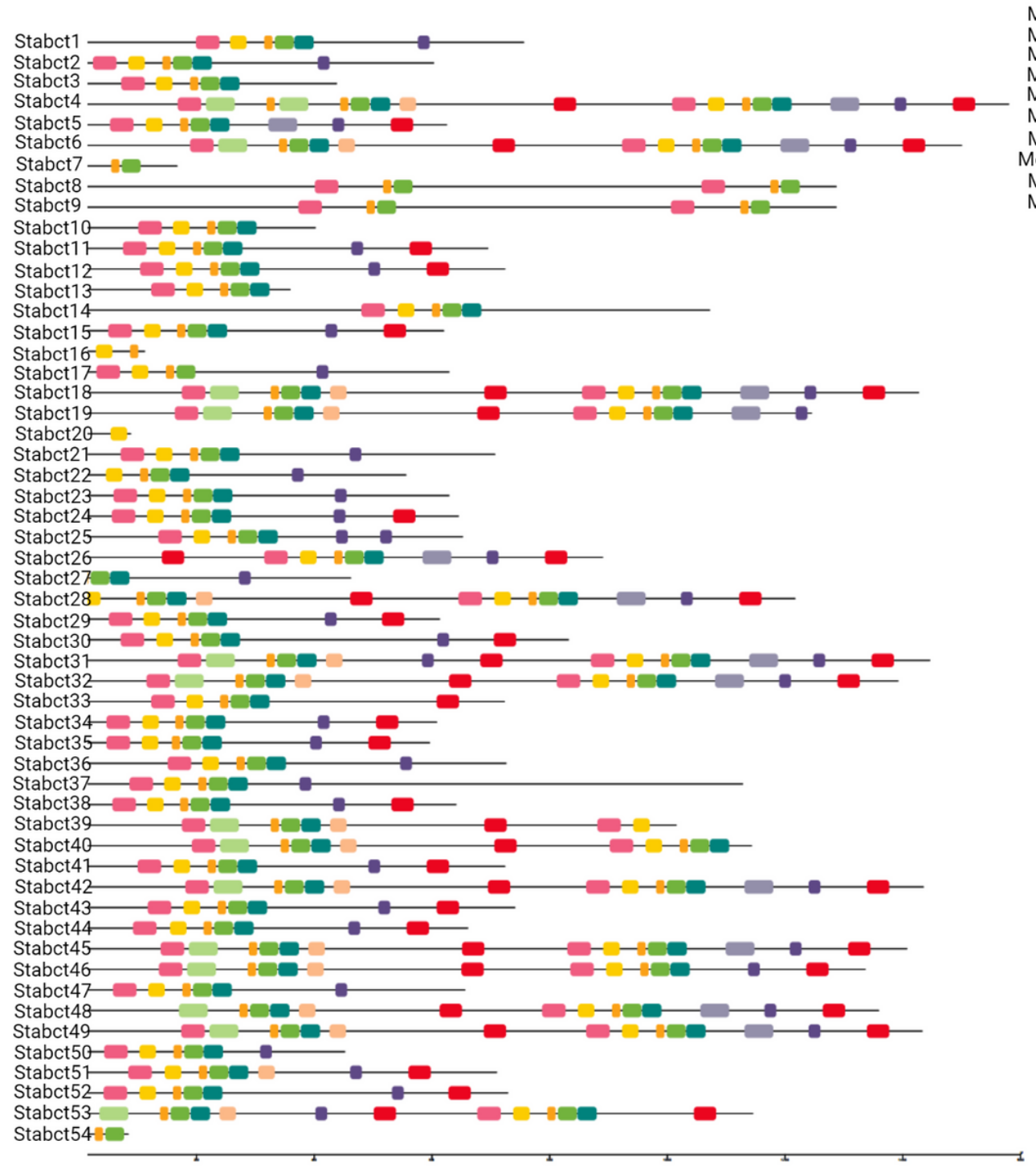

Figure 5

Cis-acting elements distribution in the regulatory regions of Stabcts 


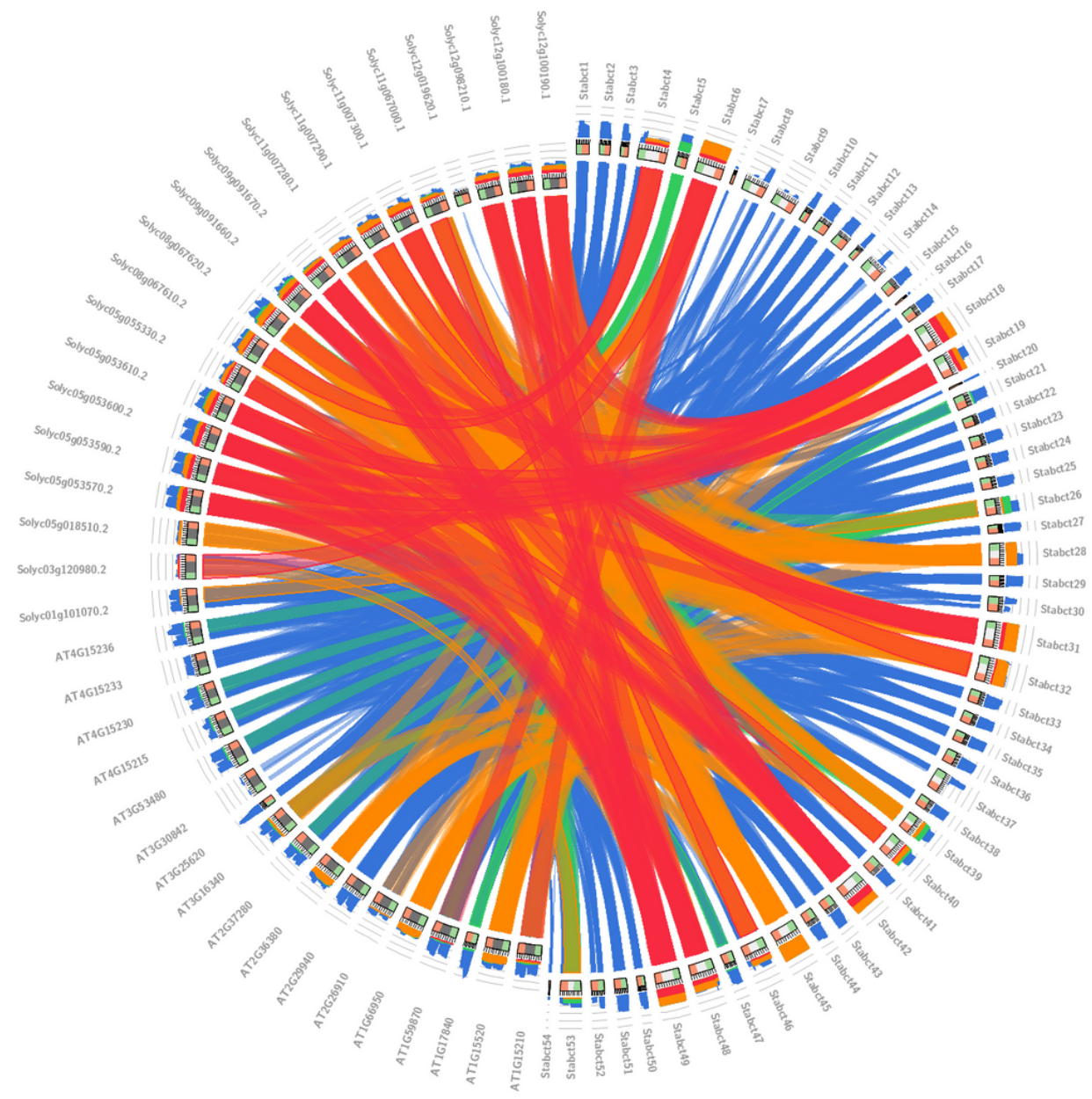

Figure 6

Synteny analysis of Stabcts among SI, St and At 

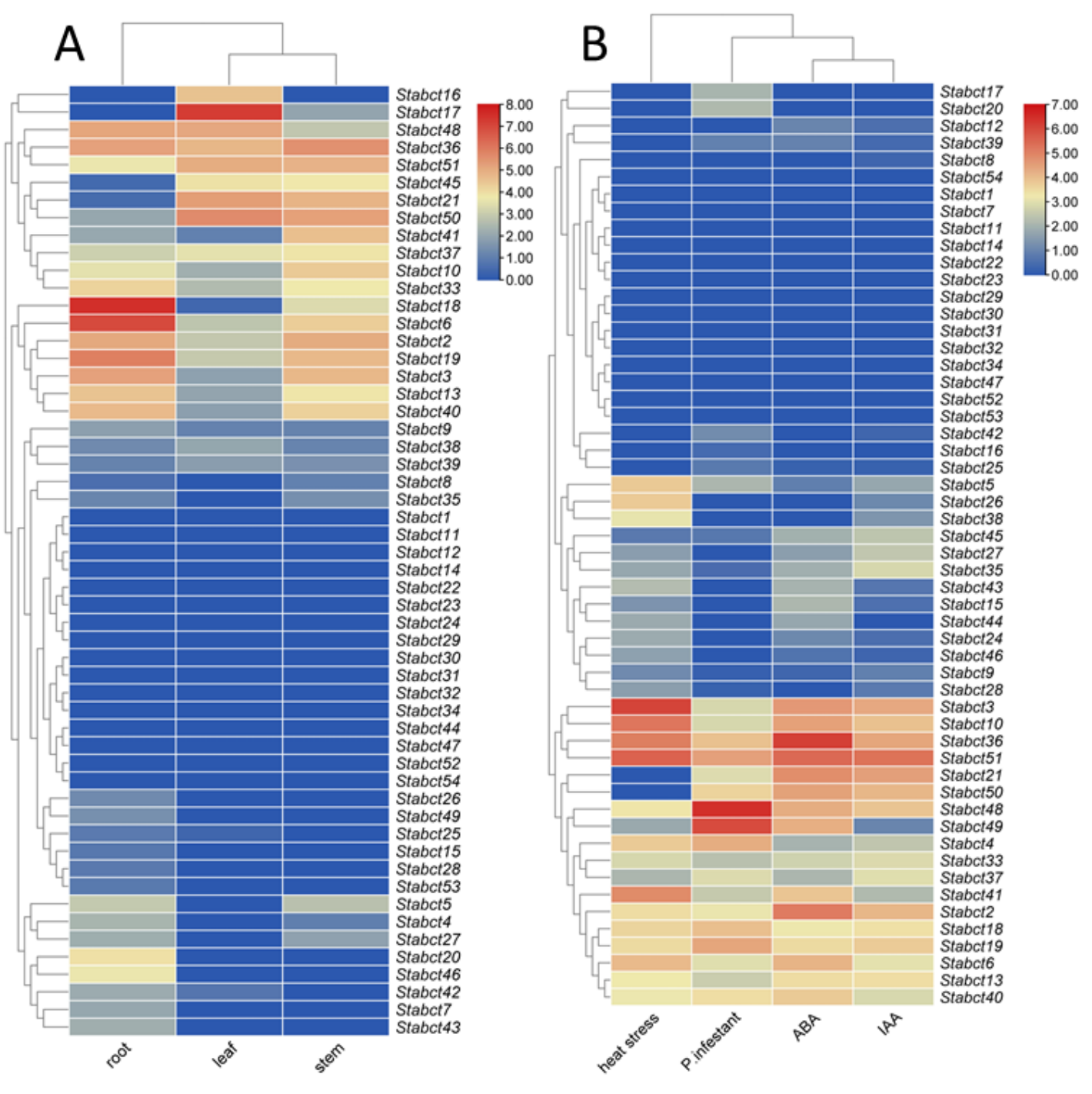

Figure 7

Expression profiling of Stabcts genes (A) leaves, root and stem (B) in response of heat, IAA, ABA and pathogen. 

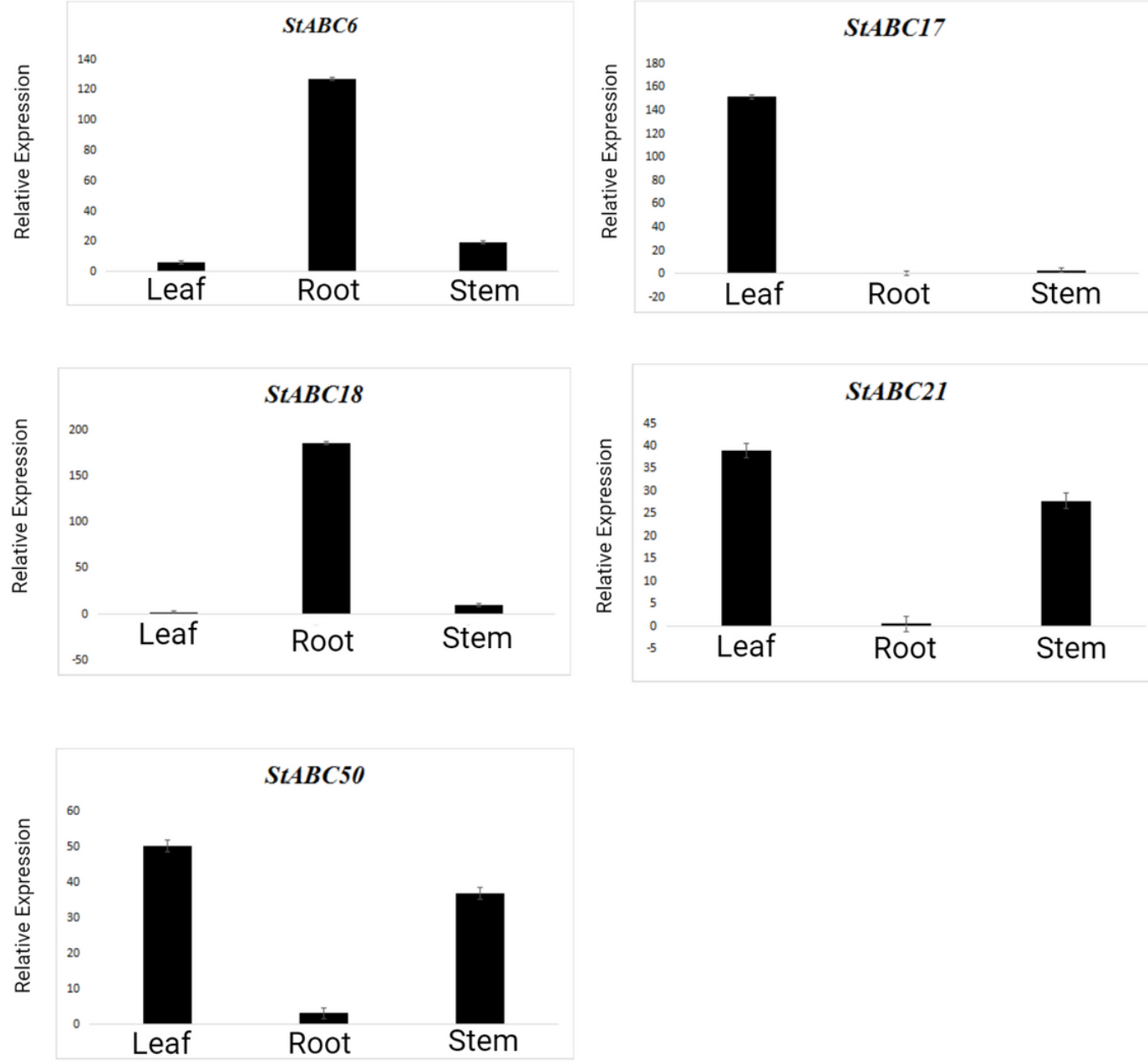

Figure 8

Real time relative expressions of Stabcts in leaves, root and stem.

\section{Supplementary Files}

This is a list of supplementary files associated with this preprint. Click to download.

- S1.xls 